\title{
A NOTE ON INVARIANT SUBSPACES FOR FINITE MAXIMAL SUBDIAGONAL ALGEBRAS
}

\author{
KICHI-SUKE SAITO
}

\begin{abstract}
Let $M$ be a von Neumann algebra with a faithful, normal, tracial state $\tau$ and $H^{\infty}$ be a finite, maximal, subdiagonal algebra of $M$. Every left- (or right-) invariant subspace with respect to $H^{\infty}$ in the noncommutative Lebesgue space $L^{p}(M, \tau), 1<p<\infty$, is the closure of the space of bounded elements it contains.
\end{abstract}

1. Introduction. Let $M$ be a von Neumann algebra with a faithful, normal, tracial state $\tau$ and let $H^{\infty}$ be a finite, maximal, subdiagonal algebra in $M$. Such algebras were defined and first studied by Arveson [1] as noncommutative analogues of weak-*Dirichlet algebras. Since the introduction of these algebras, a number of authors have investigated the structure of the invariant subspaces for $H^{\infty}$ acting on the noncommutative Lebesgue space $L^{p}(M, \tau)$ (see, in particular, [3], [5], [6], [7] and [8]). In [6], we showed that, if $\mathfrak{M}$ is a left- (or right-) invariant subspace of $L^{p}(M, \tau), 1 \leqslant p<\infty$, then $\mathfrak{M} \cap M$ contains elements different from zero. In this note, we shall show that, if $\mathfrak{M}$ is a left- (or right-) invariant subspace of $L^{p}(M, \tau), 1 \leqslant p<\infty$, then $\mathfrak{M}$ is the $L^{p}$-norm closure of $\mathfrak{M} \cap M$. The method is based on a factorization theorem, i.e. if $k$ is in $M$ with (possibly unbounded) inverse lying in $L^{2}(M, \tau)$, then there are unitary operators $u_{1}, u_{2}$ in $M$ and operators $a_{1}, a_{2}$ in $H^{\infty}$ with inverses lying in $H^{2}$ such that $k=u_{1} a_{1}=a_{2} u_{2}$.

2. Let $M$ be a von Neumann algebra with a faithful, normal, tracial state $\tau$. We shall denote the noncommutative Lebesgue spaces associated with $M$ and $\tau$ by $L^{p}(M, \tau), 1 \leqslant p<\infty$ ([2], [9]). As is customary, $M$ will be identified with $L^{\infty}(M, \tau)$. The closure of a subset $S$ of $L^{p}(M, \tau)$ in the $L^{p}$-norm $\|x\|_{p}=\tau\left(|x|^{p}\right)^{1 / p}$ will be denoted by $[S]_{p}$.

Definition. Let $H^{\infty}$ be a $\sigma$-weakly closed subalgebra of $M$ containing the identity operator 1 and let $\Phi$ be a faithful, normal expectation from $M$ onto $D=H^{\infty} \cap H^{\infty^{*}}\left(H^{\infty^{*}}=\left\{x^{*}: x \in H^{\infty}\right\}\right)$. Then $H^{\infty}$ is called a finite, maximal, subdiagonal algebra in $M$ with respect to $\Phi$ and $\tau$ in case the following conditions are satisfied: (1) $H^{\infty}+H^{\infty^{*}}$ is $\sigma$-weakly dense in $M$; (2) $\Phi(x y)=\Phi(x) \Phi(y)$, for all $x, y \in H^{\infty}$; (3) $H^{\infty}$ is maximal among those subalgebras of $M$ satisfying (1) and (2); and (4) $\tau \circ \Phi=\tau$.

For $1 \leqslant p<\infty$, the closure of $H^{\infty}$ in $L^{p}(M, \tau)$ is denoted by $H^{p}$ and the

Received by the editors December 7, 1978.

AMS (MOS) subject classifications (1970). Primary 46L15, 46L10.

Key words and phrases. Subdiagonal algebras, invariant subspaces, von Neumann algebras. 
closure of $H_{0}^{\infty}=\left\{x \in H^{\infty} ; \Phi(x)=0\right\}$ is denoted by $H_{0}^{p}$.

In [6], McAsey, Muhly and the author proved that if $k$ is in $M$ with inverse lying in $L^{2}(M, \tau)$, then there are unitary operators $u_{1}, u_{2}$ in $M$ and operators $a_{1}, a_{2}$ in $H^{\infty}$ such that $k=u_{1} a_{1}=a_{2} u_{2}$. We shall show that in fact it is possible to choose $a_{1}$ and $a_{2}$ to have inverses lying in $H^{2}$.

Proposition 1 (CF. [6, Proposition 1.2]). If $k \in M$ and $k^{-1} \in L^{2}(M, \tau)$, then there are unitary operators $u_{1}, u_{2} \in M$ and operators $a_{1}, a_{2} \in H^{\infty}$ such that $k=u_{1} a_{1}=a_{2} u_{2}$ and $a_{1}^{-1}, a_{2}^{-1} \in H^{2}$.

To Proposition 1, we need the following lemma.

LemMA 1. Suppose that $k \in M$ and $k^{-1} \in L^{2}(M, \tau)$. Then

(i) $k \notin\left[k H_{0}^{\infty}\right]_{2}$.

(ii) Let $\eta$ be the projection of $k$ on $\left[k H_{0}^{\infty}\right]_{2}$ and $\zeta=k-\eta$. Then there exists a unitary operator $u$ in $M$ such that $u \zeta \in[D]_{2},[u \zeta D]_{2}=[D]_{2}$ and $u k \in H^{\infty}$.

Proof. See proof of [6, Proposition 1.2].

Proof of Proposition 1. Keep the notations in Lemma 1. Put $a=u k$. To prove Proposition 1 , it is sufficient to prove that $a^{-1} \in H^{2}$. If $P$ is the orthogonal projection of $L^{2}(M, \tau)$ onto $[D]_{2}$, then the restriction of $P$ to $M$ equals $\Phi$. Since $\eta \in\left[k H_{0}^{\infty}\right]_{2}$, there exists a sequence $\left\{b_{n}\right\}_{n=1}^{\infty}$ in $H_{0}^{\infty}$ such that $\lim \left\|\eta-k b_{n}\right\|_{2}=0$. Then we have $u \zeta=u(k-\eta)=\lim \left(u k-u k b_{n}\right)=\lim (a$ $\left.-a b_{n}\right)$. Since $u \zeta \in[D]_{2}$ and $a b_{n} \in H_{0}^{\infty}, u \zeta=P u \zeta=\lim P\left(a-a b_{n}\right)=$ $\lim \Phi\left(a-a b_{n}\right)=\Phi(a)$. It is immediate from this that $u \zeta \in D$. Since $[u \zeta D]_{2}$ $=[D]_{2}$ by Lemma 1 and $a^{-1}=k^{-1} u^{*}$, we have for every $d \in D$,

$$
\begin{aligned}
\tau\left(\Phi(a) P\left(a^{-1}\right) u \zeta d\right) & =\tau\left(P\left(a^{-1}\right) u \zeta d \Phi(a)\right)=\tau\left(a^{-1} u \zeta d \Phi(a)\right) \\
& =\tau\left(k^{-1} \zeta d \Phi(a)\right)=\lim \tau\left(k^{-1}\left(k-k b_{n}\right) d \Phi(a)\right) \\
& =\lim \tau\left(\left(1-b_{n}\right) d \Phi(a)\right)=\tau(d \Phi(a))=\tau(u \zeta d) .
\end{aligned}
$$

Consequently we have $\Phi(a) P\left(a^{-1}\right)=1$ and so $a^{-1}=k^{-1} \zeta P\left(a^{-1}\right)$. For every $d \in D$ and every $x \in H_{0}^{\infty}$, we have $\tau\left(k^{-1} \zeta d x\right)=\lim \tau\left(k^{-1}\left(k-k b_{n}\right) d x\right)=$ $\lim \tau\left(\left(1-b_{n}\right) d x\right)=0$. Since $P\left(a^{-1}\right) \in[D]_{2}$, there exists a sequence $\left\{d_{n}\right\}_{n=1}^{\infty}$ in $D$ such that $\lim \left\|d_{n}-P\left(a^{-1}\right)\right\|_{2}=0$. Hence, for every $x \in H_{0}^{\infty}, \tau\left(a^{-1} x\right)=$ $\tau\left(k^{-1} \zeta P\left(a^{-1}\right) x\right)=\lim \tau\left(k^{-1} \zeta d_{n} x\right)=0$. Since $L^{2}(M, \tau)=H^{2} \oplus H_{0}^{2^{*}}\left(H_{0}^{2^{*}}=\right.$ $\left.\left\{x^{*}: x \in H_{0}^{2}\right\}\right)$ by $\left[1\right.$, p. 583] or [6, Proposition 1.1], we have $a^{-1} \in H^{2}$. This completes the proof. Q.E.D.

3. In this section, we collect several important facts about $H^{p}$ and $H_{0}^{p}$.

LEMMA 2. $H^{1} \cap L^{2}(M, \tau)=H^{2}$ and $H_{0}^{1} \cap L^{2}(M, \tau)=H_{0}^{2}$.

Proof. Since $L^{2}(M, \tau)=H^{2} \oplus H_{0}^{2^{*}}=H_{0}^{2} \oplus H^{2^{*}}$, this lemma is trivial.

Lemma 3. $H^{1}=\left\{x \in L^{1}(M, \tau): \tau(x y)=0\right.$ for all $\left.y \in H_{0}^{\infty}\right\}$. 
Proof. That $H^{1}$ is included in the set indicated above is clear. Conversely let $x \in L^{1}(M, \tau)$ satisfy $\tau(x y)=0, y \in H_{0}^{\infty}$. Let $x=\left|x^{*}\right| v$ be the polar decomposition of $x$. Let $f$ be the function on $[0, \infty)$ defined by the formula $f(t)=1,0<t \leqslant 1, f(t)=1 / t, t>1$, and define $k$ to be $f\left(\left|x^{*}\right|^{1 / 2}\right)$ through the functional calculus. Then note that $k \in M$ and $k^{-1} \in L^{2}(M, \tau)$. By Proposition 1, we may choose a unitary operator $u$ in $M$ and an operator $a \in H^{\infty}$ such that $k=u a$ and $a^{-1} \in H^{2}$. Then $a x$ is a nonzero element in $L^{2}(M, \tau)$. Since $L^{2}(M, \tau)=H^{2} \oplus H_{0}^{2 *}$, we have $a x \in H^{2}$ and so $x=a^{-1} a x$ $\in H^{2} H^{2} \subset H^{1}$. This completes the proof.

Since $\|\Phi(x)\|_{1} \leqslant\|x\|_{1}$ for any $x$ in $M, \Phi$ extends uniquely to a projection of norm one of $L^{1}(M, \tau)$ onto $[D]_{1}$ and we denote this extension of $\Phi$ to $L^{1}(M, \tau)$ by $\Phi$ too. Then we have the following lemma.

LEMMA 4.

$$
\begin{aligned}
H_{0}^{1} & =\left\{x \in L^{1}(M, \tau): \tau(x y)=0 \text { for all } y \in H^{\infty}\right\} \\
& =\left\{x \in H^{1}: \Phi(x)=0\right\} .
\end{aligned}
$$

Proof. The inclusion $H_{0}^{1} \subseteq\left\{x \in L^{1}(M, \tau): \tau(x y)=0\right.$ for all $\left.y \in H^{\infty}\right\}$ is clear. Now we consider any $x \in L^{1}(M, \tau)$ such that $\tau(x y)=0, y \in H^{\infty}$. Since $D \subset H^{\infty}$, we have $\tau(x y)=\tau(\Phi(x) y)=0, y \in D$, and so $\Phi(x)=0$. By Lemma 3, $x \in H^{1}$. Next we suppose $x \in H^{1}$ satisfies the equation $\Phi(x)=0$. Then there exist $x_{n} \in H^{\infty}$ such that $\left\|x_{n}-x\right\|_{1} \rightarrow 0$. Note that $\| x_{n}-\Phi\left(x_{n}\right)-$ $x \|_{1} \rightarrow 0$ and $x_{n}-\Phi\left(x_{n}\right) \in H_{0}^{\infty}$. It follows that $x \in H_{0}^{1}$. This completes the proof.

Proposition 2. Let $1 \leqslant p \leqslant \infty$.

(1) $H^{1} \cap L^{p}(M, \tau)=H^{p}$ and $H_{0}^{1} \cap L^{p}(M, \tau)=H_{0}^{p}$.

(2) $H^{p}=\left\{x \in L^{p}(M, \tau): \tau(x y)=0\right.$ for all $\left.y \in H_{0}^{\infty}\right\}$.

(3) $H_{0}^{p}=\left\{x \in L^{p}(M, \tau): \tau(x y)=0\right.$ for all $\left.y \in H^{\infty}\right\}$.

Proof. We knew already that this lemma is true for $p=2$ and for $p=\infty$ (cf. [1, Corollary 2.2.4]).

(1) We shall prove the lemma for $1<p<2$ using Proposition 1 and for $p>2$ by a duality argument.

Let $1<p<2$. Define the number $r$ by $1 / r+1 / 2=1 / p$. It is evident that $H^{p} \subseteq H^{1} \cap L^{p}(M, \tau)$. To show the reverse inclusion, consider any $x \in H^{1}$ $\cap L^{p}(M, \tau)$. Let $x=\left|x^{*}\right| v$ be the polar decomposition of $x$. Put $k=$ $f\left(\left|x^{*}\right|^{p / 2}\right)$, where $f$ is the function in the proof of Lemma 3. Then there is an element $a \in H^{\infty}$ with inverse lying in $H^{2}$ such that $a x(\neq 0) \in H^{1} \cap$ $L^{r}(M, \tau)$. Since $L^{r}(M, \tau) \subset L^{2}(M, \tau)$, we have

$$
a x \in H^{1} \cap L^{r}(M, \tau) \subset H^{1} \cap L^{2}(M, \tau)=H^{2} \subset H^{p} .
$$

So

$$
x=a^{-1} a x \in H^{2} a x \subset\left[H^{\infty} a x\right]_{p} \subset H^{p} .
$$


It follows that $H^{p}=H^{1} \cap L^{p}(M, \tau)$ in this case. $H_{0}^{p}=H_{0}^{1} \cap L^{p}(M, \tau)$ in the case $1<p<2$ may be proved in just the same way.

Let $2<p<\infty$. Here again the inclusion $H^{p} \subset H^{1} \cap L^{p}(M, \tau)$ is trivial. It is sufficient to show that if $y \in L^{q}(M, \tau)$ where $1 / p+1 / q=1$ and $y \perp H^{p}$, i.e. $\tau(y x)=0, x \in H^{p}$, then $y \perp H^{1} \cap L^{p}(M, \tau)$. Now the relation $y \perp H^{p}$ implies by Lemma 4 that $y \in H_{0}^{1} \cap L^{q}(M, \tau)=H_{0}^{q}$, as $1<q<2$. So there exist $y_{n} \in H_{0}^{\infty}$ such that $\left\|y_{n}-y\right\|_{q} \rightarrow 0$. This means that $0=\tau\left(y_{n} x\right) \rightarrow \tau(y x)$ for all $x \in H^{1} \cap L^{p}(M, \tau)$. So $y \perp H^{1} \cap L^{p}(M, \tau)$.

(2) and (3) are clear by (1) and Lemmas 3 and 4. This completes the proof.

4. Let $\mathfrak{M}$ be a closed subspace of $L^{p}(M, \tau)$. We shall say that $\mathfrak{M}$ is left(resp. right-) invariant if $H^{\infty} \mathfrak{M} \subseteq \mathfrak{M}$ (resp. $\mathfrak{M} H^{\infty} \subseteq \mathfrak{M}$ ). Our goal in this note is the following theorem.

THEOREM. Let $\mathfrak{M}$ be a left- (or right-) invariant subspace of $L^{p}(M, \tau)$, $1<p<\infty$. Then $\mathfrak{M}$ is the closure of the space of bounded operators it contains.

Proof. (1) Case $2 \leqslant p<\infty$. Define the number $q$ by the equation $1 / p+$ $1 / q=1$. If $[\mathfrak{M} \cap M]_{p} \subset \mathfrak{M}$, then there exist an element $\xi \in \mathfrak{M}$ and $x \in$ $L^{q}(M, \tau)$ such that $\tau(\xi x) \neq 0$ and $\tau(y x)=0$ for every $y \in[\mathfrak{R} \cap M]_{p}$. Let $\xi=\left|\xi^{*}\right| v$ be the polar decomposition of $\xi$. Since $\xi \in L^{p}(M, \tau) \subset L^{2}(M, \tau)$, we may form $k=f\left(\left|\xi^{*}\right|\right)$, where $f$ is the function in the proof of Lemma 3 . Note that $k \in M$ and $k^{-1} \in L^{P}(M, \tau) \subset L^{2}(M, \tau)$. By Proposition 1, we may choose a unitary operator $u$ in $M$ and an operator $a \in H^{\infty}$ such that $k=u a$ and $a^{-1} \in H^{2}$. By Proposition $2, a^{-1} \in L^{p}(M, \tau) \cap H^{2}=H^{p}$ and note that $a \xi$ is a nonzero element in $\mathfrak{M} \cap M$. Since $\mathfrak{M}$ is left-invariant, we have $b a \xi \in \mathfrak{M} \cap M$ for every $b \in H^{\infty}$ and so $\tau(b a \xi x)=0$. By Proposition 2, $a \xi x \in H_{0}^{p}$. Therefore $\tau(\xi x)=\tau\left(a^{-1} a \xi x\right)=0$. This is a contradiction.

(2) Case $1 \leqslant p<2$. Define the number $q$ and $r$ by the equations $1 / p+$ $1 / q=1$ and $1 / r+1 / 2=1 / p$. If $[\mathfrak{M} \cap M]_{p} \subsetneq \mathfrak{M}$, then there exist $\xi \in \mathfrak{M}$ and $x \in L^{q}(M, \tau)$ such that $\tau(\xi x) \neq 0$ and $\tau(\not y x)=0$ for every $y \in[\mathfrak{M} \cap$ $M]_{p}$. Let $\xi=\left|\xi^{*}\right| v$ be the polar decomposition of $\xi$. Put $k=f\left(\left|\xi^{*}\right|^{p / 2}\right)$, where $f$ is the function in the proof of Lemma 3. By Proposition 1, there is an element $a \in H^{\infty}$ with inverse lying in $H^{2}$ such that $a \xi(\neq 0) \in L^{r}(M, \tau) \cap \mathfrak{M} \subset$ $L^{2}(M, \tau) \cap \mathfrak{M}$. As in (1), there exists an element $b \in H^{\infty}$ with inverse lying in $H^{r}$ such that $b a \xi(\neq 0) \in \mathfrak{M} \cap M$. For every $c \in H^{\infty}$, we have $c b a \xi \in \mathfrak{M}$ $\cap M$ and so $\tau(c b a \xi x)=0$. By Proposition $2, b a \xi x \in H_{0}^{q}$. Since $(b a)^{-1}=$ $a^{-1} b^{-1} \in H^{2} H^{r} \subset H^{p}$, we have $\tau(\xi x)=\tau\left((b a)^{-1} b a \xi x\right)=0$. This is a contradiction.

This completes the proof.

\section{BIBLIOGRAPHY}

1. W. B. Arveson, Analyticity in operator algebras, Amer. J. Math. 89 (1967), 578-642.

2. J. Dixmier, Formes linéaires sur un anneaux d'opérators, Bull. Soc. Math. France 81 (1953), 9-39. 
3. N. Kamei, Simply invariant subspace theorems for antisymmetric finite subdiagonal algebras, Tôhoku Math. J. 21 (1969), 467-473.

4. S. Kawamura and J. Tomiyama, On subdiagonal algebras associated with flows in operator algebras, J. Math. Soc. Japan 29 (1977), 73-90.

5. R. I. Loebl and P. S. Muhly, Analyticity and flows in von Neumann algebras, J. Functional Analysis 29 (1978), 214-252.

6. M. McAsey, P. S. Muhly and K.-S. Saito, Non-self-adjoint crossed products (invariant subspaces and maximality), Trans. Amer. Math. Soc. 248 (1979), 381-410.

7. K.-S. Saito, The Hardy spaces associated with a periodic flow on a von Neumann algebra, Tôhoku Math. J. 29 (1977), 69-75.

8. On noncommutative Hardy spaces associated with flows on finite von Neumann algebras, Tôhoku Math. J. 29 (1977), 585-595.

9. I. E. Segal, A noncommutative extension of abstract integration, Ann. of Math. (2) 57 (1953), 401-457.

Department of Mathematics, Faculty of Science, Nigata University, Nilgata, 950-21, JAPAN 